\title{
An Axiomatic Approach to Feature Term Generalization
}

\author{
Hassan Aït-Kaci ${ }^{1}$ and Yutaka Sasaki ${ }^{2}$ \\ 1 Simon Fraser University \\ Burnaby, BC, Canada V5A 1S6, Canada \\ hak@sfu.ca \\ 2 NTT Communication Science Laboratories, NTT Corporation \\ 2-4 Hikaridai, Seika-cho, Soraku-gun, Kyoto 619-0237, Japan \\ sasaki@cslab.kecl.ntt.co.jp
}

\begin{abstract}
This paper presents a missing link between Plotkin's least general generalization formalism and generalization on the Order Sorted Feature $(O S F)$ foundation. A feature term (or $\psi$-term) is an extended logic term based on ordered sorts and is a normal form of an OSF-term. An axiomatic definition of $\psi$-term generalization is given as a set of OSF clause generalization rules and the least generality of the axiomatic definition is proven in the sense of Plotkin's least general generalization (lgg). The correctness of the definition is given on the basis of the axiomatic foundation. An operational definition of the least general generalization of clauses based on $\psi$-terms is also shown as a realization of the axiomatic definition.
\end{abstract}

\section{Introduction}

A feature term (or $\psi$-term) is an order-sorted logic term, i.e., an extended form of a logic term where functor symbols are ordered sorts. In addition, features (or attribute labels) are added to a sort as argument indicators. [1, 2,3

For example, the following two $\psi$-terms describe George and his mother as having the same last name "Bush" and $\mathrm{Al}$ and his mother as having the same last name "Gore":

$$
\begin{aligned}
\text { George }(\text { last } & \Rightarrow Y_{1}: \text { Bush }, \\
\text { mother } & \left.\Rightarrow \text { Barbara }\left(\text { last } \Rightarrow Y_{1}\right)\right), \\
\text { Al }(\text { last } & \Rightarrow Y_{2}: \text { Gore } \\
\text { mother } & \left.\Rightarrow \text { Pauline }\left(\text { last } \Rightarrow Y_{2}\right)\right) .
\end{aligned}
$$

In this example, George, Barbara, Bush, Al, Pauline, and Gore are sort symbols, while last and mother are feature symbols. The variables $Y_{1}$ and $Y_{2}$ link George's and Al's last names to their mother's last names.

A goal of a $\psi$-term generalization is to calculate (or induce) the following generic knowledge from the two examples:

$$
\begin{aligned}
\text { person }(\text { last } & \Rightarrow Y: \text { name, } \\
\text { mother } & \Rightarrow \text { person }(\text { last } \Rightarrow Y))
\end{aligned}
$$


Formally, without syntax sugaring, the schema is represented as

$$
\begin{aligned}
X: \text { person }(\text { last } & \Rightarrow Y: \text { name, } \\
\text { mother } & \Rightarrow Z: \text { person }(\text { last } \Rightarrow Y: \top))
\end{aligned}
$$

where $T$ is the universal-i.e., the most general-sort, and variable tags are systematically used for each sort.

The $\psi$-term is useful in many subjects of Artificial Intelligence (AI), most notably in Natural Language Processing (NLP). For instance, the feature term [5], equivalent to the $\psi$-term, is used to represent the syntax and semantics of natural language sentences. In case-based reasoning, feature terms are used as the data structures of cases [9] and the generalization of cases is a key towards the reuse of cases. Inductive Logic Programming (ILP) was extended to an induction (i.e., generalization) of logic programs based on $\psi$-terms [11|12].

While feature terms play an essential role in AI and NLP, there is a missing link between Plotkin's least general generalization formalism of classic logic terms and the generalization of $\psi$-terms on the basis of the OSF foundation. This paper presents the missing link.

\section{Preliminaries on $\psi$-Terms}

This section introduces $\psi$-terms on the basis of the Order-Sorted Feature (OSF) formalism [23].

\subsection{Syntax}

Definition 1 (OSF Signature) An OSF Signature is given by

$$
\Sigma_{O S F}=\langle\mathcal{S}, \preceq, \sqcap, \sqcup, \mathcal{F}\rangle \text {, s.t. : }
$$

$-\mathcal{S}$ is a set of sort symbols with the sorts $\top$ and $\perp$;

- $\preceq$ is a partial order on $\mathcal{S}$ such that $\top$ is the greatest element and $\perp$ is the least element;

$-\langle\mathcal{S}, \preceq, \sqcap, \sqcup\rangle$ is a lattice, where $s \sqcap t$ is defined as the infimum (or glb) of sorts $s$ and $t$ and $s \sqcup t$ is the supremum (or lub) of sorts $s$ and $t$;

$-\mathcal{F}$ is a set of feature symbols.

For sorts $s_{1}, s_{2} \in \mathcal{S}$, we denote $s_{1} \prec s_{2}$ iff $s_{1} \preceq s_{2}$ and $s_{1} \neq s_{2}$.

Let $\mathcal{V}$ be a countable infinite set of variables.

Definition 2 (OSF-terms) Given $\Sigma_{O S F}=\langle\mathcal{S}, \preceq, \sqcap, \sqcup, \mathcal{F}\rangle$, if $s \in \mathcal{S}$, $l_{1}, \ldots, l_{n} \in \mathcal{F}, X \in \mathcal{V}, n \geq 0$, and $t_{1}, \ldots, t_{n}$ are OSF-terms, then an OSF-term has the form

$$
X: s\left(l_{1} \Rightarrow t_{1}, \ldots, l_{n} \Rightarrow t_{n}\right)
$$


Let $\psi=X: s\left(l_{1} \Rightarrow t_{1}, \ldots, l_{n} \Rightarrow t_{n}\right) . X$ is called the root variable of $\psi$, which is described as $\operatorname{Root}(\psi)$, and $s$ is called the root sort of $\psi$, which is described as $\operatorname{Sort}(\psi)$.

For a lighter notation, hereafter we omit variables that are not shared and the sort of a variable when it is $T$.

Definition 3 ( $\psi$-terms) An OSF-term

$$
\psi=X: s\left(l_{1} \Rightarrow \psi_{1}, \ldots, l_{n} \Rightarrow \psi_{n}\right)
$$

is in a normal form (and is called a $\psi$-term) if:

- For any variable $V_{i}$ in $\psi, V_{i}$ is the root variable of at most one non-top $\psi$-term, i.e., one whose root sort is not $\top$;

- $s$ is a nonbottom sort in $\mathcal{S}$;

$-l_{1}, \ldots, l_{n}$ are pairwise distinct feature symbols in $\mathcal{F}$;

$-\psi_{1}, \ldots, \psi_{n}$ are $\psi$-terms.

We will see that OSF-terms can be normalized to $\psi$-terms by OSF clause normalization rules, which are given in Section 2.3. or are otherwise proven to be inconsistent by being reduced to $\perp$.

Let $\psi=X: s\left(l_{1} \Rightarrow \psi_{1}, \ldots, l_{n} \Rightarrow \psi_{n}\right) . s\left(l_{1} \Rightarrow \psi_{1}, \ldots, l_{n} \Rightarrow \psi_{n}\right)$ is called an untagged $\psi$-term.

Definition 4 (Feature Projection) Given a $\psi$-term $t=X: s\left(l_{1} \Rightarrow t_{1}, \ldots\right.$, $l_{n} \Rightarrow t_{n}$ ), the $l_{i}$ projection of $t$ (written as $t . l_{i}$ ) is defined as $t . l_{i}=t_{i}$.

The definitions of atoms, literals, clauses, Horn clauses, and definite clauses are as usual with the difference being that terms are $\psi$-terms. If a feature is a non-zero integer $1, \ldots, n$, then a $\psi$-term $X: s\left(1 \Rightarrow t_{1}, 2 \Rightarrow t_{2}, \ldots, n \Rightarrow t_{n}\right)$ can be abbreviated to $X: s\left(t_{1}, t_{2}, \ldots, t_{n}\right)$.

\subsection{Semantics}

Definition 5 (OSF Algebras) An OSF Algebra is a structure $\mathcal{A}=\left\langle D^{\mathcal{A}},\left(s^{\mathcal{A}}\right)_{s \in \mathcal{S}},\left(l^{\mathcal{A}}\right)_{l \in \mathcal{F}}\right\rangle$ s.t. :

- $D^{\mathcal{A}}$ is a non-empty set, called a domain of $\mathcal{A}$;

- for each sort symbol $s \in \mathcal{S}, s^{\mathcal{A}} \subseteq D^{\mathcal{A}}$; in particular, $\top^{\mathcal{A}}=D^{\mathcal{A}}$ and $\perp^{\mathcal{A}}=\emptyset$;

$-\left(s \sqcap s^{\prime}\right)^{\mathcal{A}}=s^{\mathcal{A}} \cap s^{\mathcal{A}}$ for two sorts $s, s^{\prime} \in \mathcal{S}$;

$-\left(s \sqcup s^{\prime}\right)^{\mathcal{A}}=s^{\mathcal{A}} \cup s^{\prime \mathcal{A}}$ for two sorts $s, s^{\prime} \in \mathcal{S}$;

- for each feature symbol $l \in \mathcal{F}, l^{\mathcal{A}}: D^{\mathcal{A}} \rightarrow D^{\mathcal{A}}$.

Definition 6 ( $\mathcal{A}$-Valuation) Given $\Sigma_{O S F}=\langle\mathcal{S}, \preceq, \sqcap, \sqcup, \mathcal{F}\rangle$, an $\mathcal{A}$-valuation is a function $\alpha: \mathcal{V} \rightarrow D^{\mathcal{A}}$. 
Definition 7 (Term Denotation) Let $t$ be a $\psi$-term of the form

$$
t=X: s\left(l_{1} \Rightarrow t_{1}, \ldots, l_{n} \Rightarrow t_{n}\right)
$$

Given an OSF Algebra $\mathcal{A}$ and an $\mathcal{A}$-valuation $\alpha$, the term denotation of $t$ is given by

$$
\begin{gathered}
\llbracket t \rrbracket^{\mathcal{A}, \alpha}=\{\alpha(X)\} \cap s^{\mathcal{A}} \cap \bigcap_{1 \leq i \leq n}\left(l_{i}^{\mathcal{A}}\right)^{-1}\left(\llbracket t_{i} \rrbracket^{\mathcal{A}, \alpha}\right) . \\
\llbracket t \rrbracket^{\mathcal{A}}=\bigcup_{\alpha: \mathcal{V} \rightarrow D^{\mathcal{A}}} \llbracket t \rrbracket^{\mathcal{A}, \alpha} .
\end{gathered}
$$

\section{$2.3 \quad$ Unification of $\psi$-Terms}

An alternative syntactic presentation of the information conveyed by OSF-terms can be translated into a constraint clause [2].

Definition 8 (OSF-Constraints) An order-sorted feature constraint (OSFconstraint) has one of the following forms:

$-X: s$

$-X \doteq Y$

$-X . l \doteq Y$

where $X$ and $Y$ are variables in $\mathcal{V}, s$ is a sort in $\mathcal{S}$, and $l$ is a feature in $\mathcal{F}$.

Definition 9 (OSF-clauses) An OSF-clause $\phi_{1} \& \ldots \& \phi_{\eta}$ is a finite, possibly empty conjunction of OSF-constraints $\phi_{1}, \ldots, \phi_{n}(n \geq 0)$. 1

We can associate an OSF-term with a corresponding OSF-clause.

Let $\psi$ be a $\psi$-term of the form

$$
\psi=X: s\left(l_{1} \Rightarrow \psi_{1}, \ldots, l_{n} \Rightarrow \psi_{n}\right)
$$

An OSF-clause $\phi(\psi)$ corresponding to an OSF-term $\psi$ has the following form:

$$
\begin{gathered}
\phi(\psi)=X: s \& X . l_{1} \doteq X_{1}^{\prime} \& \ldots \& X . l_{n} \doteq X_{n}^{\prime} \\
\& \phi\left(\psi_{1}\right) \quad \& \ldots \& \phi\left(\psi_{n}\right),
\end{gathered}
$$

where $X, X_{1}^{\prime}, \ldots, X_{n}^{\prime}$ are the root variables of $\psi, \psi_{1}, \ldots, \psi_{n}$, respectively. We say $\phi(\psi)$ is dissolved from the OSF-term $\psi$.

Example 1 Let $\left.\psi=X: s\left(l_{1} \Rightarrow Y: t, l_{2} \Rightarrow Y: \top\right)\right)$. The OSF-clause of $\psi$ is: $\phi(\psi)=X: s \quad \& X . l_{1} \doteq Y \& Y: t \quad \& X . l_{2} \doteq Y \& Y: \top$

\footnotetext{
${ }^{1}$ We sometimes regard an OSF-clause as a set of OSF constraints.
} 
Sort Intersection:

$$
\frac{\phi \& X: s \& X: s^{\prime}}{\phi \& X: s \sqcap s^{\prime}}
$$

Inconsistent Sort:

$$
\frac{\phi \& X: \perp}{X: \perp}
$$

Variable Elimination:

$$
\begin{gathered}
\frac{\phi \& X \doteq X^{\prime}}{\phi\left[X / X^{\prime}\right] \& \quad X \doteq X^{\prime}} \\
\text { if } X \neq X^{\prime} \text { and } X \in \operatorname{Var}(\phi)
\end{gathered}
$$

Feature Decomposition:

$$
\begin{array}{ccc}
\phi \quad \& \quad X . l \doteq X^{\prime} \quad \& \quad X . l \doteq X^{\prime \prime} \\
\hline \phi \quad \& \quad X . l \doteq X^{\prime} \quad \& \quad X^{\prime} \doteq X^{\prime \prime}
\end{array}
$$

Fig. 1. OSF Clause Normalization Rules

On the other hand, an OSF-clause $\phi$ can be converted to an OSF-term $\psi(\phi)$ as follows: first complete it by adding as many $V: \top$ constraints as needed so that there is exactly one sort constraint for every occurrence of a variable $V$ in an $X . l=V$ constraint, where $X$ is a variable and $l$ is a feature symbol; then convert it by the following $\psi$ transform:

$$
\psi(\phi)=X: s\left(l_{1} \Rightarrow \psi\left(\phi\left(Y_{1}\right)\right), \ldots, l_{n} \Rightarrow \psi\left(\phi\left(Y_{n}\right)\right)\right)
$$

where $X$ is a root variable of $\phi, \phi$ contains $X: s$, and $X . l_{1} \doteq Y_{1}, \ldots, X . l_{n} \doteq Y_{n}$ are all of the other constraints in $\phi$ with an occurrence of variable $X$ on the left-hand side. $\phi(Y)$ denotes the maximal subclause of $\phi$ rooted by $Y$.

Definition 10 (Solved OSF-Constraint) An OSF-clause $\phi$ is called solved if for every variable $X, \phi$ contains:

- at most one sort constraint of the form $X: s$, with $\perp \prec s$;

- at most one feature constraint of the form $X . l \doteq Y$ for each X.l;

- no equality constraint of the form $X \doteq Y$.

Given $\phi$ in a normal form, we will refer to its part in a solved form as $\operatorname{Solved}(\phi)$.

Example 2 Let $\phi=X: s \& X . l_{1} \doteq Y \& Y: t \& X . l_{2} \doteq Y \& Y: \top$. The solved normal form of $\phi$ is :

Solved $(\phi)=X: s \& X . l_{1} \doteq Y \& Y: t \& X . l_{2} \doteq Y$.

Theorem 1 [2] The rules of Fig. 1] are solution-preserving, finite-terminating, and confluent (modulo variable renaming). Furthermore, they always result in a normal form that is either an inconsistent OSF clause or an OSF clause in a solved form together with a conjunction of equality constraints.

Note that $\operatorname{Var}(\phi)$ is the set of variables occurring in an OSF-clause $\phi$ and $\phi[X / Y]$ stands for the OSF-clause obtained from $\phi$ after replacing all occurrences of $Y$ by $X$. 
Sort Induction (SI):

$$
\begin{gathered}
\frac{\left\{X_{1} \backslash X\right\} \cup \Gamma_{1},\left\{X_{2} \backslash X\right\} \cup \Gamma_{2} \vdash \phi \&\left(\left(X_{1}: s_{1} \& \phi_{1}\right) \vee\left(X_{2}: s_{2} \& \phi_{2}\right)\right)}{\left\{X_{1} \backslash X\right\} \cup \Gamma_{1},\left\{X_{2} \backslash X\right\} \cup \Gamma_{2} \vdash \phi \&\left(X: s_{1} \sqcup s_{2}\right) \&\left(\left(X_{1}: s_{1} \& \phi_{1}\right) \vee\left(X_{2}: s_{2} \& \phi_{2}\right)\right)} \\
\text { if } \neg \exists s(X: s \in \phi)
\end{gathered}
$$

Feature Induction (FI):

$$
\begin{gathered}
\frac{\left\{X_{1} \backslash X\right\} \cup \Gamma_{1},\left\{X_{2} \backslash X\right\} \cup \Gamma_{2} \vdash \phi \&\left(\left(X_{1} . l \doteq Y_{1} \& \phi_{1}\right) \vee\left(X_{2} . l \doteq Y_{2} \& \phi_{2}\right)\right)}{\left\{X_{1} \backslash X, Y_{1} \backslash Y\right\} \cup \Gamma_{1},\left\{X_{2} \backslash X, Y_{2} \backslash Y\right\} \cup \Gamma_{2} \vdash \phi \& X . l \doteq Y \&\left(\left(X_{1} . l \doteq Y_{1} \& \phi_{1}\right) \vee\left(X_{2} . l \doteq Y_{2} \& \phi_{2}\right)\right)} \\
i f \neg \exists y\left(Y_{1} \backslash y \in\left\{X_{1} \backslash X\right\} \cup \Gamma_{1} \text { and } Y_{2} \backslash y \in\left\{X_{2} \backslash X\right\} \cup \Gamma_{2}\right)
\end{gathered}
$$

Coreference Induction (CI):

$$
\begin{gathered}
\frac{\left\{X_{1} \backslash X, Y_{1} \backslash Y\right\} \cup \Gamma_{1},\left\{X_{2} \backslash X, Y_{2} \backslash Y\right\} \cup \Gamma_{2} \vdash \phi \&\left(\left(X_{1} . l \doteq Y_{1} \& \phi_{1}\right) \vee\left(X_{2} . l \doteq Y_{2} \& \phi_{2}\right)\right)}{\left\{X_{1} \backslash X, Y_{1} \backslash Y\right\} \cup \Gamma_{1},\left\{X_{2} \backslash X, Y_{2} \backslash Y\right\} \cup \Gamma_{2} \vdash \phi \& X . l \doteq Y \&\left(\left(X_{1} . l \doteq Y_{1} \& \phi_{1}\right) \vee\left(X_{2} . l \doteq Y_{2} \& \phi_{2}\right)\right)} \\
\text { if } X . l \doteq Y \notin \phi
\end{gathered}
$$

Fig. 2. OSF Clause Generalization Rules

Theorem 2 ( $\psi$-term Unification) [2] Let $\psi_{1}$ and $\psi_{2}$ be two $\psi$-terms. Let $\phi$ be the normal form of the OSF-clause $\phi\left(\psi_{1}\right) \& \phi\left(\psi_{2}\right) \& X_{1} \doteq X_{2}$, where $X_{1}$ and $X_{2}$ are the root variables of $\psi_{1}$ and $\psi_{2}$, respectively. Then, $\phi$ is an inconsistent clause iff the glb of the two $\psi$-terms is $\perp$. If $\phi$ is not an inconsistent clause, then the glb $\psi_{1} \sqcap \psi_{2}$ is given by the normal OSF-term $\psi(\operatorname{Solved}(\phi))$.

\section{Axiomatic $\psi$-Term Generalization}

As a dual of $\psi$-term unification, $\psi$-term generalization (or anti-unification) can be defined as OSF clause generalization rules.

To define the generalization, we introduce a new constraint symbol $\vee$, where $A \vee B$ denotes the generalization of two OSF clauses $A$ and $B$.

A $\psi$-term generalization rule is of the form:

$$
\frac{\Gamma_{1}, \Gamma_{2} \vdash \phi \&\left(\phi_{1} \vee \phi_{2}\right)}{\Gamma_{1}^{\prime}, \Gamma_{2}^{\prime} \vdash \phi^{\prime} \&\left(\begin{array}{lll}
\phi_{1} & \vee & \phi_{2}
\end{array}\right)}
$$

where $\Gamma_{1}$ and $\Gamma_{2}$ are sets of substitutions of the form $\left\{X_{1} \backslash X_{1}^{\prime}, \ldots, X_{n} \backslash X_{n}^{\prime}\right\}, 2$, $\phi$ and $\phi^{\prime}$ are OSF-clauses, and $\phi_{1}$ and $\phi_{2}$ are solved normal forms of OSF-clauses of target $\psi$-terms $\psi_{1}$ and $\psi_{2}$, respectively.

Definition 11 (Axiomatic Generalization) Let $\phi_{1}$ and $\phi_{2}$ be solved normal forms of $\psi_{1}$ and $\psi_{2}$, respectively, and $\Gamma_{1}$ and $\Gamma_{2}$ be variable substitutions. Then, a generalized OSF-clause $\phi$ of

$$
\Gamma_{1}, \Gamma_{2} \vdash \phi \&\left(\phi_{1} \vee \phi_{2}\right)
$$

$\overline{2}$ This means that $X_{i}^{\prime}$ is substituted by $X_{i}$. 
is obtained by applying OSF clause generalization rules (Fig. 2) until no rule is applicable, initiated with

$$
\left\{X_{1} \backslash X\right\},\left\{X_{2} \backslash X\right\} \vdash\left(\phi_{1} \vee \phi_{2}\right)
$$

where $X_{1}=\operatorname{Root}\left(\psi_{2}\right), X_{2}=\operatorname{Root}\left(\psi_{2}\right)$, and $X$ is a fresh variable.

A generalized $\psi$-term is given as $\psi(\phi)$.

Proposition 1 The result of the axiomatic generalization is an OSF-clause in the normal form.

Proposition 2 The OSF clause generalization is finite terminating.

Proof. Termination follows from the fact that the number of variables in $\phi_{1}$ is finite because OSF-clauses are finite, and each of the three rules SI, FI, and CI strictly decreases the number of combinations of variables in $\phi_{1}$ and $\phi_{2}$ that satisfy the preconditions of the OSF generalization rules.

From the definition of $\phi(\cdot)$ and OSF clause normalization rules (Fig. 1), the number of variables in $\phi_{i}$ is finite since $\psi_{i}$ is finite by the definition of OSFterms. The Sort Induction (SI) strictly decreases the number of variable pairs that satisfy the conditions of the generalization rules. That is, the variable pair $X_{1}$ of $X_{1}: s_{1}$ and $X_{2}$ of $X_{2}: s_{2}$ does not satisfy the precondition of SI after its application. The Feature Induction (FI) strictly decreases the number of pairs of variable pairs that satisfy the rule conditions. The pair of variable pairs $\left\langle X_{1}, Y_{1}\right\rangle$ and $\left\langle X_{2}, Y_{2}\right\rangle$ does not satisfy the precondition of FI after its application. Since FI is only applicable a finite number of times, FI increases the finite number of pairs applicable to the SI and CI rules. Like FI, the Coreference Induction (CI) strictly decreases the number of pairs of variable pairs that satisfy the rule conditions. The pair of variable pairs $\left\langle X_{1}, Y_{1}\right\rangle$ and $\left\langle X_{2}, Y_{2}\right\rangle$ does not satisfy the precondition of CI after its application.

\subsection{Least General Generalization}

This section newly introduces the least general generalization of $\psi$-terms along the line of Plotkin's least general generalization (lgg) [10].

Definition 12 (Sorted Substitution) A sorted substitution has the form $\left\{X_{1}: s_{1} / Y_{1}: t_{1}, \ldots, X_{n}: s_{n} / Y_{n}: t_{n}\right\}$, where $X_{1}, \ldots, X_{n}$ are pairwise distinct variables and $Y_{1}, \ldots, Y_{n}$ are variables in $\mathcal{V}, s_{1}, \ldots, s_{n}$ and $t_{1}, \ldots, t_{n}$ are sort symbols with $\perp \prec s_{i} \preceq t_{i}$ for every $i$. If expression $E$ is a term, a literal, or a clause, $E \theta$ is the result of replacing all occurrences of $Y_{i}: t_{i}$ by $X_{i}: s_{i}$ and $Y_{i}$ by $X_{i}$ simultaneously for every $i$.

Note that the sorted substitution changes only variable names and sorts; it does not add or remove constraints of the form $X . l \doteq Y$. This means that the sorted substitution preserves the structure of an original expression. 
Definition 13 (Sorted Ordering of $\psi$-terms) Let $\psi_{1}$ and $\psi_{2}$ be two $\psi$ terms. Let $\phi_{1}$ and $\phi_{2}$ be solved normal forms of OSF clauses of $\psi_{1}$ and $\psi_{2}$, respectively. $\psi_{1} \leq \psi_{2}$ iff there exists a sorted substitution $\theta$ such that $\phi_{1} \theta \subseteq \phi_{2}$ 3 and $\left(\operatorname{Root}\left(\psi_{2}\right): \operatorname{Sort}\left(\psi_{2}\right) / \operatorname{Root}\left(\psi_{1}\right): \operatorname{Sort}\left(\psi_{1}\right)\right) \in \theta$.

We read $\psi_{1} \leq \psi_{2}$ as meaning that $\psi_{1}$ is more general than $\psi_{2}$.

Example $3(X: s) \leq Y: t(l \Rightarrow Z: u)$ with $t \preceq s$ because for $\theta=\{Y: t / X: s\},(X: s) \theta$ $=\{Y: t\} \subseteq(Y: t \& Y . l \doteq Z \& Z: u)$.

Proposition 3 If $\phi$ is the result of the $\psi$-term generalization of the OSF-clauses of $\psi$-terms $\psi_{1}$ and $\psi_{2}$ and $\psi=\psi(\phi)$, then $\psi \leq \psi_{1}$ and $\psi \leq \psi_{2}$ in terms of sorted ordering $\leq$.

Proof. Prove $\psi \leq \psi_{1}$. Let $\phi_{1}$ be a solved normal form of $\psi_{1}$. Let the final result of the $\psi$-term generalization be $\Gamma_{1}, \Gamma_{2} \vdash \phi \&\left(\phi_{1} \vee \phi_{2}\right)$ with $\Gamma_{1}=$ $\left\{X_{1}^{\prime} \backslash X_{1}, \ldots, X_{n}^{\prime} \backslash X_{n}\right\}$. Let $s_{i}^{\prime}$ be the sort of $X_{i}^{\prime}: s_{i}^{\prime} \in \phi\left(\psi_{1}\right)$ and $s_{i}$ be the sort of $X_{i}: s_{i} \in \phi(\psi)$. A sorted substitution $\theta=\left\{X_{1}^{\prime}: s_{1}^{\prime} / X_{1}: s_{1}, \ldots, X_{n}^{\prime}: s_{n}^{\prime} / X_{n}: s_{n}\right\}$ clearly satisfies the relation $\phi \theta \subseteq \phi_{1}$ according to the OSF generalization rules. The proof of $\psi \leq \psi_{2}$ is the same.

Definition 14 (Least General Generalization) Let $\psi_{1}$ and $\psi_{2}$ be $\psi$-terms. $\psi$ is the least general generalization (lgg) of $\psi_{1}$ and $\psi_{2}$ iff

(1) $\psi \leq \psi_{1}$ and $\psi \leq \psi_{2}$.

(2) If $\psi^{\prime} \leq \psi_{1}$ and $\psi^{\prime} \leq \psi_{2}$, then $\psi^{\prime} \leq \psi$.

Theorem 3 (Least Generality of Generalization) The axiomatic $\psi$-term generalization is a least general generalization with respect to the sorted ordering of $\psi$-terms.

Proof. (1) $\psi \leq \psi_{1}$ and $\psi \leq \psi_{2}$ are immediate from Proposition 3. (2) Let $\psi_{1}$ and $\psi_{2}$ be $\psi$-terms and $\psi$ be the result of the $\psi$-term generalization of $\psi_{1}$ and $\psi_{2}$. Also assume that there exists a $\psi$-term $\psi^{\prime}$ such that $\psi^{\prime} \leq \psi_{1}, \psi^{\prime} \leq \psi_{2}$, and $\psi<\psi^{\prime}$, i.e., $\psi$ is strictly more general than $\psi^{\prime}$. Let $\phi, \phi_{1}, \phi_{2}$, and $\phi^{\prime}$ be solved normal forms of $\psi, \psi_{1}, \psi_{2}$, and $\psi^{\prime}$, respectively. The assumption $\psi<\psi^{\prime}$ requires that there be an OSF constraint $C^{\prime}$ in $\phi^{\prime}$ such that no sorted substitution $\theta$ satisfies $C^{\prime} \theta \in \phi$ and $\phi^{\prime} \theta \subseteq \phi$. There are two cases to be considered: (case 1) $C^{\prime}$ is of the form $X^{\prime}: s^{\prime}$ and (case 2) $C^{\prime}$ is of the form $X^{\prime} . l \doteq Y^{\prime}$.

Case 1: From the assumptions $\psi^{\prime} \leq \psi_{1}$ and $\psi^{\prime} \leq \psi_{2}, X^{\prime}: s^{\prime}$ can be substituted to $X_{1}: s_{1}$ in $\phi_{1}$ and $X_{2}: s_{2}$ in $\phi_{2}$. Therefore, $s_{1} \preceq s^{\prime}$ and $s_{2} \preceq s^{\prime}$. Since sorted substitutions preserve the structures of $\psi$-terms, according to $\psi$-term generalization rules, if $X_{1}: s_{1}$ and $X_{2}: s_{2}$ correspond to the same constraint $X^{\prime}: s$, then $X: s$ should be included in $\phi$. By SI, sort $s$ in $\psi$ is the least upper

\footnotetext{
${ }^{3}$ We regard a clause as a set of constraints here.
} 
bound (lub) of a sort $s_{1}$ in $\psi_{1}$ and a sort $s_{2}$ in $\psi_{2}$. This contradicts $s_{1} \preceq s^{\prime}$, $s_{2} \preceq s^{\prime}$, and $s^{\prime} \prec s$.

Case 2: Similarly, from the assumptions of $\psi^{\prime} \leq \psi_{1}$ and $\psi^{\prime} \leq \psi_{2}, X^{\prime} . l \doteq$ $Y^{\prime}$ can be substituted to $X_{1} .1 \doteq Y_{1}$ in $\phi_{1}$ and $X_{2} . l \doteq Y_{2}$ in $\phi_{2}$. Since sorted substitutions preserve the structures of $\psi$-terms, if $X_{1} . l \doteq Y_{1}$ and $X_{2} . l \doteq Y_{2}$ correspond to the same constraint $X^{\prime} . l \doteq Y^{\prime}$, then $X . l \doteq Y$ should be in $\phi$. This is a contradiction.

\section{Operational $\psi$-Term Generalization}

On the other hand, an operational definition of $\psi$-term generalization [11] has been defined as an extension of Plotkin's least general generalization (lgg) using the following notations. $a$ and $b$ represent untagged $\psi$-terms. $s, t$, and $u$ represent $\psi$-terms. $f, g$, and $h$ represent sorts. $X, Y$, and $Z$ represent variables in $\mathcal{V}$.

Definition 15 (lgg of $\psi$-terms) Let $\psi_{1}$ and $\psi_{2}$ be $\psi$-terms. $\operatorname{lgg}\left(\psi_{1}, \psi_{2}\right)$ is defined as follows with the initial history Hist $=\{\}$.

1. $\operatorname{lgg}(X: a, X: a)=X: a$.

2. $\operatorname{lgg}(X: a, Y: b)=Z: \top$, where $X \neq Y$ and the tuple $(X, Y, Z)$ is already in the history Hist.

3. If $s=X: f\left(l_{1}^{s} \Rightarrow s_{1}, \ldots, l_{n}^{s} \Rightarrow s_{n}\right)$ and $t=Y: g\left(l_{1}^{t} \Rightarrow t_{1}, \ldots, l_{m}^{t} \Rightarrow t_{m}\right)$, then $\operatorname{lgg}(s, t)=Z:(f \sqcup g)\left(l_{1} \Rightarrow \operatorname{lgg}\left(s \rightarrow l_{1}, t \rightarrow l_{1}\right), \ldots, l_{|L|} \Rightarrow \operatorname{lgg}\left(s \rightarrow l_{|L|}, t \rightarrow\right.\right.$ $\left.\left.l_{|L|}\right)\right)$, where $l_{i} \in L=\left\{l_{1}^{s}, \ldots, l_{n}^{s}\right\} \cap\left\{l_{1}^{t}, \ldots, l_{m}^{t}\right\}$. Then, $(X, Y, Z)$ is added to Hist.

Note that in this definition $s \rightarrow l$ is defined as $s \rightarrow l=X: a$ if $s . l=X: \top$ and $X: a \in \psi_{1}$ with $a \neq \top$ else $s \rightarrow l=$ s.l. $t \rightarrow l$ is defined similarly.

For example, the $\operatorname{lgg}$ of $X$ :passenger $\left(\right.$ of $\left.\Rightarrow X^{\prime}: 10\right)$ and $Y: \operatorname{man}\left(o f \Rightarrow Y^{\prime}: 2\right)$ is $Z$ : person(of $\Rightarrow Z^{\prime}:$ number $)$, if passenger $\sqcup$ man $=$ person and $10 \sqcup 2=$ number.

Theorem 4 (Correctness) The result of the operational $\psi$-term generalization $\psi$ is the least general generalization of $\psi$-terms $\psi_{1}$ and $\psi_{2}$ in terms of the sorted ordering.

Proof. (Sketch) Each step of the operational definition can be translated into OSF generalization rules. Step 1 is a special case of Sort Induction.

Step 2 is Coreference Induction where tuple $(\mathrm{X}, \mathrm{Y}, \mathrm{Z})$ in Hist corresponds to $X \backslash Z$ in $\Gamma_{1}$ and $Y \backslash Z$ in $\Gamma_{2}$.

Step 3 is Sort Induction of $X: f \sqcup g$ and Feature Induction, where tuple $(X, Y, Z)$ added to Hist corresponds to $X \backslash Z$ and $Y \backslash Z$ which are added to variable substitutions. All of the steps of the operational definition are realizations of the OSF clause generalization. Therefore, the result of the operational generalization is a least general generalization of $\psi$-terms. 


\section{Generalization of Clauses Based on $\psi$-Terms}

This section presents the least general generalization of logic programs based on $\psi$-terms along the line of Plotkin's lgg of atoms and clauses [10].

Definition 16 (Ordering of Atoms) Let $A_{1}=p\left(\psi_{1}, \ldots, \psi_{n}\right)$ and $A_{2}=$ $q\left(\psi_{1}^{\prime}, \ldots, \psi_{n}^{\prime}\right)$ be atomic formulae based on $\psi$-terms. $A_{1} \leq A_{2}$ iff $A_{1} \theta=A_{2}$ for some sorted substitution $\theta$ which includes a substitution replacing the root variable of $\psi_{i}$ by the root variable of $\psi_{i}^{\prime}$.

Definition 17 (Ordering of Clauses) Let $C_{1}$ and $C_{2}$ be clauses based on $\psi$ terms. $C_{1} \leq C_{2}$ iff $C_{1} \theta \subseteq C_{2}$ for some sorted substitution $\theta$ which includes substitutions replacing the root variables of $\psi$-terms in $C_{1}$ by the corresponding root variables of $\psi$-terms in $C_{2}$.

Definition 18 (Lgg of Atoms) Given a signature $\Sigma_{O S F}=\langle\mathcal{S}, \preceq, \sqcap, \sqcup, \mathcal{F}\rangle$ and a set of predicate symbols $\mathcal{P}$, let $P$ and $Q$ be atomic formulae. An operational definition of a function $\operatorname{lgg}(P, Q)$ that computes the least general generalization of $P$ and $Q$ is as follows.

1. If $P=p\left(s_{1}, \ldots, s_{n}\right)$ and $Q=p\left(t_{1}, \ldots, t_{n}\right)$,

$\lg g(P, Q)=p\left(\operatorname{lgg}\left(s_{1}, t_{1}\right), \ldots, \operatorname{lgg}\left(s_{n}, t_{n}\right)\right)$ with the sharing of history Hist.

2. Otherwise, $\operatorname{lgg}(P, Q)$ is undefined.

Definition 19 (Lgg of Literals) Let $P$ and $Q$ be atoms and $L_{1}$ and $L_{2}$ be literals. The lgg of the literals is defined as follows [8].

1. If $L_{1}$ and $L_{2}$ are atoms, then $\lg g\left(L_{1}, L_{2}\right)$ is the lgg of the atoms.

2. If $L_{1}$ and $L_{2}$ are of the forms $\neg P$ and $\neg Q$, respectively, then $\operatorname{lgg}\left(L_{1}, L_{2}\right)=$ $\operatorname{lgg}(\neg P, \neg Q)=\neg \operatorname{lgg}(P, Q)$.

3. Otherwise, $\lg \left(L_{1}, L_{2}\right)$ is undefined.

Definition 20 (Lgg of Clauses) Let clauses $C=\left\{L_{1}, \ldots, L_{n}\right\}$ and $D=$ $\left\{K_{1}, \ldots, K_{m}\right\}$. Then $\lg g(C, D)=\left\{\operatorname{lgg}\left(L_{i}, K_{j}\right) \mid L_{i} \in C, K_{j} \in D\right.$ and $\lg g\left(L_{i}, K_{j}\right)$ is not undefined\}.

The least general generality of lggs of atoms, literals, and clauses is conservative extension of Plotkin's lgg since the operational $\psi$-term generalization is an $\operatorname{lgg}$ of terms.

\section{Related Works}

The definition of the least general generalization (lgg) was first investigated in [10]. The lgg of $\psi$-terms has already been illustrated [1]; however, axiomatic and operational definitions have been left untouched. The lgg of a subset of description logics, called the least common subsumer (LCS), was studied in [4]. The lgg of feature terms, which are equivalent to $\psi$-terms, can be found in [9]. The generalization for Sorted First Order Predicate Calculus (SFOPC) [7] is presented in [6]. 


\section{Conclusion and Remarks}

Two generalization approaches have been presented and related. An axiomatic definition of $\psi$-term generalization was presented as $\psi$-term generalization rules. The definition was proven to be a least general generalization (lgg) in terms of Plotkin's lgg on the OSF foundation. The correctness of an operational definition of $\psi$-term generalization was provided on the basis of the generalization rules. The operational definition was shown to be one realization of the axiomatic generalization. An lgg of clauses based on $\psi$-terms was presented, and a fundamental bridge between $\psi$-term generalization and the lgg useful for inductive logic programming was given. The main benefit of this paper is that it expresses generalization (and hence induction) as an OSF constraint construction process. This approach may lead to other axiomatic constraint systems provided with inductive algorithms.

Acknowledgment. This paper is based on the research results done during the first author's stay at the NTT Communication Science Laboratories, Kyoto, Japan, in April, 1999.

\section{References}

1. Hassan Aït-Kaci and Roger Nasr. LOGIN: A logic programming language with built-in inheritance. Journal of Logic Programming, 3:185-215, 1986.

2. Hassan Aït-Kaci and Andreas Podelski. Towards a meaning of LIFE. Logic Program., 16(3-4):195-234, July-August 1993.

3. Hassan Aït-Kaci, Andreas Podelski, and Seth Copen Goldstein. Order-sorted feature theory unification. Journal of Logic Programming, 30(2):99-124, 1997.

4. Franz Baader, Ralf Küsters and Ralf Molitor. Computing Least Common Subsumers in Description Logics with Existential Restrictions. Proccedings of the Sixteenth International Joint Conference on Artificial Intelligence, pages 96-101, 1999.

5. Bob Carpenter. The Logic of Typed Feature Structures, volume 32 of Cambridge Tracts in Theoretical Computer Science. Cambridge University Press, Cambridge, UK, 1992.

6. Alan M. Frisch and C. David Page Jr. Generalization with taxonomic information. In Proceedings of the 8th National Conference on Artificial Intelligence, pages 755761, Boston, MA, 1990. AAAI-90.

7. Alan M. Frisch. A general framework for sorted deduction: Fundamental results on hybrid reasoning. In Proceedings of the 1st International Conference on Principles of Knowledge Representation and Reasoning, pages 126-136, 1989.

8. Nada Lavrač and Sašo Džeroski. Inductive Logic Programming: Techniques and Applications. Ellis Horwood, 1994.

9. Enric Plaza. Cases as terms: A feature term approach to the structured representation of cases. In Proceedings of the 1st International Conference on Case-Based Reasoning, pages 263-27, 1995.

10. Gordon Plotkin. A note on inductive generalization. In Machine Intelligence, pages 153-163. Edinburgh University Press, 1969. 
11. Yutaka Sasaki. Induction of logic programs based on $\psi$-terms. In Proceedings of the 10th International Conference on Algorithmic Learning Theory, pages 169-181, Tokyo, Japan, 1999. ALT-99, Springer-Verlag LNAI 1720.

12. Yutaka Sasaki. Hierarchically Sorted Inductive Logic Programming and Its Application to Information Extraction. Ph.D thesis, Graduate School of Systems and Information Engineering, University of Tsukuba, Japan, September 2000.

\section{Appendix (Example of Axiomatic $\psi$-Term Generalization)}

Suppose that we have two $\psi$-terms $\psi_{1}$ and $\psi_{2}$, and $u=s \sqcup t$.

$$
\begin{aligned}
& \psi_{1}=X: s(a \Rightarrow Z: s, b \Rightarrow Z) \\
& \psi_{2}=Y: t(a \Rightarrow W: t, b \Rightarrow U: t)
\end{aligned}
$$

The normal form of OSF clauses of these $\psi$-terms are:

$$
\begin{aligned}
& \phi\left(\psi_{1}\right)=X: s \& X . a \doteq Z \& Z: s \& X . b \doteq Z \\
& \phi\left(\psi_{2}\right)=Y: t \& Y . a \doteq W \& W: t \& Y . b \doteq U \& U: t .
\end{aligned}
$$

A generalization of these two OSF clauses is obtained by applying generalization rules to the OSF clause $C=\phi\left(\psi_{1}\right) \vee \phi\left(\psi_{2}\right)$. The following steps show the process to achieve a generalization.

$\{X \backslash V\},\{Y \backslash V\} \vdash$

$((\underline{X: s} \& X . a \doteq Z \& Z: s \& X . b \doteq Z) \vee$

$(\underline{Y: t} \& Y . a \doteq W \& W: t \& Y . b \doteq U \& U: t))$

$\{X \backslash V\},\{Y \backslash V\} \vdash \quad(V: u)$

$\& \quad((X: s \& X . a \doteq Z \& Z: s \& X . b \doteq Z) \vee$

$(Y: t \& \underline{Y . a \doteq W} \& W: t \& Y . b \doteq U \& U: t))$ (by SI)

$\left\{X \backslash V, Z \backslash V^{\prime}\right\},\left\{Y \backslash V, W \backslash V^{\prime}\right\} \vdash \quad\left(V: u \& V . a \doteq V^{\prime}\right)$

$\& \quad((X: s \& X . a \doteq Z \& \underline{Z: s} \& X . b \doteq Z) \vee$

$(Y: t \& Y . a \doteq W \& \underline{W: t} \& Y . b \doteq U \& U: t))($ by $\mathrm{FI})$

$\left\{X \backslash V, Z \backslash V^{\prime}\right\},\left\{Y \backslash V, \overline{W \backslash V^{\prime}}\right\} \vdash \quad\left(V: u \& V . a \doteq V^{\prime} \& V^{\prime}: u\right)$

$\&((X: s \& X . a \doteq Z \& Z: s \& X . b \doteq Z) \vee$

$(Y: t \& Y . a \doteq W \& W: t \& \underline{Y . b \doteq U} \& U: t))$ (by SI)

$\left\{X \backslash V, Z \backslash V^{\prime}, Z \backslash V^{\prime \prime}\right\},\left\{Y \backslash V, W \backslash V^{\prime}, U \backslash V^{\prime \prime}\right\} \vdash\left(V: u \& V . a \doteq V^{\prime} \& V^{\prime}: u \& V . b \doteq V^{\prime \prime}\right)$

$\&((\underline{X: s} \& X . a \doteq Z \& Z: s \& X . b \doteq Z) \vee$

$(Y: t \& Y . a \doteq W \& W: t \& Y . b \doteq U \& \underline{U: t}))($ by $\mathrm{FI})$

$\left\{X \backslash V, Z \backslash V^{\prime}, Z \backslash V^{\prime \prime}\right\},\left\{Y \backslash V, W \backslash V^{\prime}, U \backslash V^{\prime \prime}\right\} \vdash$

$\left(\underline{\left.V: u \& V . a \doteq V^{\prime} \& V^{\prime}: u \& V . b \doteq V^{\prime \prime} \& V^{\prime \prime}: u\right)}\right.$

$\&((X: s \& X . a \doteq Z \& Z: s \& X . b \doteq Z) \vee$

$(Y: t \& Y . a \doteq W \& W: t \& Y . b \doteq U \& U: t))$ (by SI)

Therefore, an OSF clause $\psi_{3}$ of a $\psi$-term generalization of $\psi_{1}$ and $\psi_{2}$ is:

$$
\phi_{3}=V: u \& V . a \doteq V^{\prime} \& V^{\prime}: u \& V . b \doteq V^{\prime \prime} \& V^{\prime \prime}: u
$$

The $\psi$-term of $\psi_{3}$ is: $\psi\left(\phi_{3}\right)=V: u\left(a \Rightarrow V^{\prime}: u, b \Rightarrow V^{\prime \prime}: u\right)$. 